\title{
A SPECTRAL COMMUTANT LIFTING THEOREM
}

\author{
HARI BERCOVICI, CIPRIAN FOIAS, AND ALLEN TANNENBAUM
}

\begin{abstract}
The commutant lifting theorem of [24] may be regarded as a very general interpolation theorem from which a number of classical interpolation results may be deduced. In this paper we prove a spectral version of the commutant lifting theorem in which one bounds the spectral radius of the interpolant and not the norm. We relate this to a spectral analogue of classical matricial Nevanlinna-Pick interpolation.
\end{abstract}

\section{INTRODUCTION}

Interpolation by bounded analytic functions in the disc is a topic with a rich mathematical history and wide-ranging applications. Indeed, here the areas of complex analysis, operator theory, and hyperbolic geometry intersect creating a rich synthesis and a research subject with its own flavor and techniques. This paper is concerned with a new twist to the subject in which we consider interpolation theory not with analytic matrices of bounded norm, but with bounded spectral radius.

In order to put our results in proper perspective, let us briefly review classical Nevanlinna-Pick theory $[16,19,20]$. Accordingly, we are given $2 n$ points $z_{1}, \ldots, z_{n}, w_{1}, \ldots, z_{n} \in D$ (the open unit disc), and we would like to find necessary and sufficient conditions for the existence of an analytic function $f: D \rightarrow \bar{D}$ such that $f\left(z_{j}\right)=w_{j}$ for $1 \leq j \leq n$. Already when $n=2$, the Schwarz-Pick lemma [3] gives a nontrivial obstruction. This basically amounts to the fact that analytic functions on the disc must be contractive relative to the Poincare (hyperbolic) metric. Using these ideas a necessary and sufficient condition can be written for the existence of $f$ in terms of the positivity of a certain Hermitian matrix (called appropriately the "Nevanlinna-Pick" matrix).

Received by the editors May 8, 1989. The results of this paper were presented at the 97 th Annual Meeting of the AMS, January 17-20, 1990, in the Special Session "Linear operators, matrix functions, and control" organized by J. A. Ball and I. Gohberg..

1980 Mathematics Subject Classification (1985 Revision). Primary 47A20; Secondary 47Bxx, 30E05, $93 \mathrm{C} 05$.

Key words and phrases. Commutant lifting theorem, dilation theory, spectral radius, interpolation theory.

This work was supported in part by grants from the Research Fund of Indiana University, Department of Energy DE-FG02-86ER25020, National Science Foundation DMS-8858149, DMS8521683, DMS-8802596, ECS-8704047, DMS-8811084, and by the Air Force Office of Scientific Research AFOSR-88-0020 and AFOSR-90-0024. 
More precisely, when the $z_{j}$ are distinct, $f$ exists if and only if

$$
\left[\frac{1-w_{j} \bar{w}_{k}}{1-z_{j} \bar{z}_{k}}\right]_{1 \leq j, k \leq n} \geq 0 .
$$

The classical approach to such interpolation problems (including the general Carathéodory-Fejér problem [1] in which one is allowed to interpolate with finite multiplicities) is complex analytic. A remarkable fact discovered by Donald Sarason [22] is that many results on interpolation by bounded analytic functions may be deduced in an operator theoretic manner, and using operator theory one can even extend interpolation theory to cover interpolation with points with infinite multiplicity. (Sarason called his result "generalized interpolation in $H^{\infty}$ ".) Sarason's theorem was significantly broadened by the Sz.-Nagy-Foias commutant lifting theorem $[24,25]$ which allows one to even interpolate on the disc by bounded analytic operator-valued functions. Since these seminal papers, there has been much work done on interpolation theory from an operator-theoretic point of view, in particular in a series of classic papers by Adamjan-Arov-Krein $[1,2]$, and the theory of Ball-Helton [4]. See also the monograph [5] for an exposition of results in this area.

Besides the intrinsic mathematical beauty of the aforementioned work on interpolation by bounded analytic functions, these results have had a major impact in a number of areas of applied science, e.g., control and systems theory, signal processing, geophysics, circuit theory, and biomedical engineering. For details see $[13,14,15,17,26]$, and the references therein. The specific problem we will be considering in this paper had precisely such an applied origin, namely that in robust system design, i.e., the design of feedback control systems in the presence of parameter uncertainty [7]. However, we believe that the mathematical problem which arises from this has a purely intrinsic operator-theoretic interest, and motivates a completely new type of interpolation theory with a number of novel twists.

In order to describe our result, let us consider the Nevanlinna-Pick theory in the matrix case. As above, let $z_{j} \in D$ be distinct, $1 \leq j \leq n$, and let $F_{1}, \ldots, F_{n}$ be $N \times N$ matrices. Then we are interested in finding necessary and sufficient conditions for the existence of an analytic (in the disc) $N \times N$ matrix-valued function $F(z)$ with $F\left(z_{j}\right)=F_{j}(1 \leq j \leq n)$, and such that $\|F\| \leq 1$. The existence of $F$ can again be reduced to the determination of the positivity of a certain Nevanlinna-Pick matrix. (This fact can be deduced, e.g., from the commutant lifting theorem [24, 25]. See also the discussion in $\S 6$ below). However, in our case we will not be interested in bounding the norm, but instead the spectral radius of the interpolating functions. We shall give necessary and sufficient conditions for the existence of an interpolating $F$ whose spectral radius is bounded by 1 . This will be deduced as a consequence of a general spectral commutant lifting result (see Theorem 3 below). 
We now summarize the contents of this paper. In $\S 2$, we give the relevant background on commutant lifting and dilation theory, and illustrate the relevance of this theory to interpolation. In $\S 3$, we define a notion of "generalized spectral radius" which will be used in the formulation of the spectral commutant lifting theorem which is proven in $\S 4$. In $\S 5$, we describe some properties of the generalized spectral radius relative to the similarity action of a certain algebraic group. In $\S 6$, we prove a spectral version of the classical matricial Nevanlinna-Pick theorem. We conclude the paper in $\S 7$, where we discuss a number of examples illuminating the theory.

We would like to thank the referee for his numerous helpful comments, and especially for the nice argument he provided us in connection to Proposition 6 below (see Remark 7).

\section{BACKGROUND ON THE COMMUTANT LIFTING THEOREM}

Throughout this paper $\mathscr{H}$ will denote a complex separable Hilbert space. By "operator" we shall always mean "bounded linear operator", unless explicitly stated otherwise. We let $B(\mathscr{H})$ denote the set of operators on $\mathscr{H}$.

In this section, we shall give some background on the commutant lifting theorem [22, 24, 25] and its connection to interpolation theory. Full details about the results described in this section can be found in the aforementioned references as well as [5].

We first begin with a key result due to Sz.-Nagy [23]. Let $T: \mathscr{H} \rightarrow \mathscr{H}$ be a contraction, i.e., an operator such that $\|T\| \leq 1$. Then in [23] it is proven that there exists an isometry $U$ on a Hilbert space $\mathscr{K}$ such that

$$
\mathscr{H}=\bigvee_{n=0}^{\infty} U^{n} \mathscr{H}
$$

and $P_{\mathscr{H}} U=T P_{\mathscr{H}}$, where $P_{\mathscr{H}}: \mathscr{K} \rightarrow \mathscr{H}$ denotes orthogonal projection. $U$ is called the minimal isometric dilation of $T$. Then the commutant lifting theorem (proved in a special case in [22], and in complete generality in [24]) may be stated as follows:

Theorem 1 (Commutant lifting theorem). Let $\mathscr{H}$ and $\mathscr{H}^{\prime}$ denote (complex separable) Hilbert spaces with $T: \mathscr{H} \rightarrow \mathscr{H}$, and $T^{\prime}: \mathscr{H}^{\prime} \rightarrow \mathscr{H}^{\prime}$ contractions. Let $A: \mathscr{H} \rightarrow \mathscr{H}^{\prime}$ be an intertwining contraction for $T$ and $T^{\prime}$, i.e., $A T=T^{\prime} A$. Let $U: \mathscr{K} \rightarrow \mathscr{K}$ and $U^{\prime}: \mathscr{K}^{\prime} \rightarrow \mathscr{K}^{\prime}$ denote the minimal isometric dilations of $T$ and $T^{\prime}$ respectively. Then there exists a contraction $\widehat{A}: \mathscr{K} \rightarrow \mathscr{K}^{\prime}$ such that $U^{\prime} \hat{A}=\widehat{A} U$ and $P_{\mathscr{H}^{\prime}} \hat{A}=A P_{\mathscr{H}}$, where $P_{\mathscr{H}^{\prime}}: \mathscr{K}^{\prime} \rightarrow \mathscr{H}^{\prime}$ and $P_{\mathscr{H}}: \mathscr{K} \rightarrow \mathscr{H}$ denote orthogonal projections.

Remarks 1. (i) $\widehat{A}$ is called an intertwining dilation of $A$.

(ii) For the commutant lifting theorem we do not need the minimal isometric dilation, but any isometric dilation of $T$ satisfying $T^{*}=U^{*} \mid \mathscr{H}$. Moreover, in the proof we may take without loss of generality $\mathscr{H}=\mathscr{H}^{\prime}$ and $T=T^{\prime}$. In 
this case, $\widehat{A}$ is called a commuting dilation of $A$. (See $[5,24,25]$, for all the details.)

The power of the commutant lifting theorem is that it allows one to derive most of the classical interpolation results involving bounded analytic functions in a unified, elegant manner even when the functions are operator-valued. In order to give the reader an idea how this is done we consider here the classical scalar Nevanlinna-Pick problem following [22].

Recall from the Introduction that the problem of Nevanlinna-Pick concerns finding necessary and sufficient conditions for the existence of an analytic $f: D$ $\rightarrow D$, such that $f\left(z_{j}\right)=w_{j}, j=1, \ldots, n$. We assume as before that the $z_{j}$ are distinct. We now put the Nevanlinna-Pick problem into the commutant lifting framework.

Accordingly, set

$$
m(z)=\prod_{i=1}^{n} \frac{z-z_{i}}{1-\overline{z_{i}} z} .
$$

Let $H(m):=H^{2} \ominus m H^{2}$. (All of the Hardy spaces in this paper will be defined in the unit disc $D$ in the standard way.) One can then prove that $H(m)$ is an $n$-dimensional vector space with basis $f_{1}, \ldots, f_{n}$, where

$$
f_{j}(z):=\left(\prod_{k \neq j} \frac{z-z_{k}}{1-\overline{z_{k}} z}\right) \frac{1}{1-\overline{z_{j}} z} \quad \text { for } j=1, \ldots, n .
$$

Now let $S: H^{2} \rightarrow H^{2}$ denote the canonical unilateral right shift defined by multiplication by $z$. For $P_{H(m)}: H^{2} \rightarrow H(m)$ orthogonal projection, set $S(m):=P_{H(m)} S \mid H(m)$ (the compressed shift). Again, it is easy to show [22] that

for $1 \leq j \leq n$.

$$
S(m) f_{j}=z_{j} f_{j}
$$

We can now define a linear operator $A: H(m) \rightarrow H(m)$ such that $A f_{j}=w_{j} f_{j}$ for $j=1, \ldots, n$. Clearly $A$ commutes with $T$. We are now ready to sketch the proof of the Nevanlinna-Pick theorem:

Theorem 2 (Nevanlinna-Pick). Assume the notation given above. Then there exists an analytic $f: D \rightarrow \bar{D}$ such that $f\left(z_{j}\right)=w_{j}$ for $j=1, \ldots, n$ if and only if the Navanlinna-Pick matrix

$$
\left[\frac{1-\overline{w_{i}} w_{j}}{1-\overline{z_{i}} z_{j}}\right]_{i, j=1, \ldots, n} \geq 0 .
$$

Proof. First of all let us note that the nonnegative definiteness of NevanlinnaPick's matrix is equivalent to the inequality $I-A^{*} A \geq 0$ or, equivalently, to $\|A\| \leq 1$. By the commutant lifting theorem, this condition is equivalent to the existence of an operator $\hat{A}$, commuting with $S$, such that $\|\widehat{A}\| \leq 1$ and

$$
A=P_{H(m)} \widehat{A} \mid H(m) \text {. }
$$


It is well known that every operator $\widehat{A}$ commuting with $S$ is given by

$$
\widehat{A} u=f u, \quad u \in H^{2},
$$

for some $f \in H^{\infty}$, and $\|\widehat{A}\|=\|f\|_{\infty}$. Thus we see that the nonnegative definiteness of the Nevanlinna-Pick matrix is equivalent to the existence of a function $f \in H^{\infty}\|f\|_{\infty} \leq 1$, such that

$$
A u=P_{H(m)} f u=f(S(m)) u, \quad u \in H(m) .
$$

This last equality simply means that $w_{j}=f\left(z_{j}\right), j=1,2, \ldots, n$. The theorem follows from these observations.

We will discuss the matrix spectral version of this result in $\S 6$.

\section{GenERALIZED SPECTRAL RADIUS}

We will define the main object of study of this paper in this section, and derive some of its basic properties. We use the notation and terminology of $\S 2$ here.

Given $T \in B(\mathscr{H})$, we let

$$
\{T\}^{\prime}:=\{A \in B(\mathscr{H}): A T=T A\} .
$$

We now come to the following key definition:

Definition 1. Let $T, A \in B(\mathscr{H})$. Then we set

$$
\rho_{T}(A):=\inf \left\{\left\|M A M^{-1}\right\|: M \text { is invertible and } M \in\{T\}^{\prime}\right\} .
$$

We call $\rho_{T}(A)$ the $T$-spectral radius of $A$.

Remarks 2. (i) Notice from a theorem of Rota [21], that we have

$$
\rho_{a I}(A)=\|A\|_{\mathrm{sp}} \quad \forall a \in \mathbf{C},
$$

where $I$ denotes the identity operator of $\mathscr{H}$, and $\|A\|_{\mathrm{sp}}$ is the spectral radius of the operator $A$. In particular, we see for any $T$,

$$
\|A\|_{\mathrm{sp}} \leq \rho_{T}(A) \leq\|A\| \text {. }
$$

(ii) One can show that the infimum defining $\rho_{T}(A)$ is not necessarily a minimum. (See Example 4 in $§ 7$.

We can now state the following proposition.

Proposition 1. (i) If $T$ is an isometry, then $\rho_{T}(A)=\|A\|_{\mathrm{sp}}$ for every $A \in\{T\}^{\prime}$.

(ii) If $T$ is normal, then $\rho_{T}(A)=\|A\|_{\mathrm{sp}}$ for every $A \in\{T\}^{\prime}$.

(iii) If $\rho_{T}(A)=\|A\|_{\mathrm{sp}}$ for every $A \in\{T\}^{\prime}$, then $\sigma(T)$ is a spectral set for $T$. In particular, if $\mathscr{H}$ is finite dimensional, then $T$ is normal.

Proof. (i) Without loss of generality, we may assume that $\|A\|_{\mathrm{sp}}<1$. Since we already know that

$$
\|A\|_{\mathrm{sp}} \leq \rho_{T}(A)
$$


it suffices to prove the opposite inequality. Equivalently, we must show that $\|A\|_{\mathrm{sp}}<1$ implies that $\rho_{T}(A) \leq 1$. In order to do this, we first choose $n>0$ so large that $\left\|A^{n}\right\|<1$. Denote by $\mathscr{H}_{1}$ the space $\mathscr{H}$ endowed with the new scalar product (with associated norm \|\|$_{1}$ ) defined by

$$
\langle h, k\rangle_{1}:=\langle h, k\rangle+\langle A h, A k\rangle+\cdots+\left\langle A^{n-1} h, A^{n-1} k\right\rangle
$$

for $h, k \in \mathscr{H}$ (where $\langle$,$\rangle denotes the inner product on \mathscr{H}$ with associated norm \|\|$)$. Denote by $A_{1}$ and $T_{1}$ the operators $A$ and $T$ regarded as operators on $\mathscr{H}_{1}$, and denote by $X: \mathscr{H} \rightarrow \mathscr{H}_{1}$ the identity. Clearly, we have

$$
\|h\|^{2} \leq\|X h\|_{1}^{2} \leq\left(1+\|A\|^{2}+\cdots+\left\|A^{n-1}\right\|^{2}\right)\|h\|^{2} \quad \forall h \in \mathscr{H},
$$

so that $X$ is invertible. Furthermore,

$$
\left\|A_{1} h\right\|_{1}^{2}=\sum_{j=1}^{n-1}\left\|A^{j} h\right\|^{2}+\left\|A^{n} h\right\|^{2} \leq \sum_{j=1}^{n-1}\left\|A^{j} h\right\|^{2}+\|h\|^{2}=\|h\|_{1}^{2},
$$

and

$$
\left\|T_{1} h\right\|_{1}^{2}=\sum_{j=1}^{n-1}\left\|A^{j} T h\right\|^{2}=\sum_{j=1}^{n-1}\left\|T A^{j} h\right\|^{2}=\sum_{j=1}^{n-1}\left\|A^{j} h\right\|^{2}=\|h\|_{1}^{2},
$$

so that $\left\|A_{1}\right\| \leq 1$ and $T_{1}$ is an isometry. Since $T_{1}=X T X^{-1}$, it follows that there exists a unitary operator $U: \mathscr{H}_{1} \rightarrow \mathscr{H}$ such that $T=U T_{1} U^{-1}$. Now define $M:=U X$ and note that $M$ is invertible, $M$ commutes with $T$, and

$$
\left\|M A M^{-1}\right\|=\left\|U X A X^{-1} U^{-1}\right\|=\left\|X A X^{-1}\right\|=\left\|A_{1}\right\| \leq 1,
$$

which completes the proof of part (i).

(ii) As in the preceding proof, it suffices to show that $\rho_{T}(A) \leq 1$ if $\|A\|_{\mathrm{sp}}<1$. Choose $n$ such that $\left\|A^{n}\right\| \leq 1$, and note that the inequality $\|A h\|_{1} \leq\|h\|_{1}, h \in$ $\mathscr{H}$, is equivalent to $\left\|B A B^{-1}\right\| \leq 1$, where $B:=\left(\sum_{j=0}^{n-1} A^{* j} A^{j}\right)^{1 / 2}$. By the Fuglede theorem, $B$ commutes with $T$, thus showing that

$$
\rho_{T}(A) \leq\left\|B A B^{-1}\right\| \leq 1,
$$

as required.

(iii) Assume that $\rho_{T}(A)=\|A\|_{\mathrm{sp}}$ for every $A \in\{T\}^{\prime}$, and let $f$ be analytic in a neighborhood of $\sigma(T)$. Clearly

$$
\|f(T)\|_{\mathrm{sp}}=\sup \{|f(\lambda)|: \lambda \in \sigma(T)\}
$$

and

$$
\begin{aligned}
\rho_{T}(f(T)) & =\inf \left\{\left\|M f(T) M^{-1}\right\|: M \in\{T\}^{\prime}, M \text { invertible }\right\} \\
& =\inf \left\{\left\|f\left(M T M^{-1}\right)\right\|: M \in\{T\}^{\prime}, M \text { invertible }\right\}=\|f(T)\|,
\end{aligned}
$$

completing the proof of the proposition.

Let $\mathscr{E}$ be a separable Hilbert space, and let $H^{2}(\mathscr{E}):=H^{2} \otimes \mathscr{E}$ be the Hilbert space of square-summable $\mathscr{E}$-valued power series (see [25]). Given a bounded 
analytic function $A: D \rightarrow B(\mathscr{E})$, we can consider the multiplication operator $M_{A}: H^{2}(\mathscr{E}) \rightarrow H^{2}(\mathscr{E})$ defined by

$$
\left(M_{A} f\right)(z):=A(z) f(z), \quad f \in H^{2}(\mathscr{E}), \quad z \in D .
$$

The operator $M_{A}$ commutes with the unilateral shift $S$ defined by

$$
(S f)(z):=z f(z), \quad f \in H^{2}(\mathscr{E}), \quad z \in D,
$$

and it satisfies the norm equality

$$
\left\|M_{A}\right\|=\|A\|_{\infty}=\sup \{\|A(z)\|: z \in D\} .
$$

We now have

Proposition 2. Let $A: \bar{D} \rightarrow B(\mathscr{E})$ be a continuous function, analytic in $D$. Then

$$
\rho_{S}(A)=\left\|M_{A}\right\|_{\mathrm{sp}}=\sup _{z \in D}\|A(z)\|_{\mathrm{sp}}=\max _{z \in \bar{D}}\|A(z)\|_{\mathrm{sp}} .
$$

Proof. Since the function $A \mapsto\|A\|_{\text {sp }}$ is upper semicontinuous, the function $z \mapsto\|A\|_{\mathrm{sp}}$ attains its maximum in $\bar{D}$. Thus we can set

$$
r:=\max \left\{\|A\|_{\mathrm{sp}}: z \in \bar{D}\right\} \text {. }
$$

Now the continuous functions

$$
f_{n}(z):=\max \left\{\left\|A(z)^{2^{n}}\right\|^{1 / 2^{n}}, r\right\}, \quad n \geq 0,
$$

decrease to $r$, and therefore by Dini's theorem, they converge uniformly. Since

$$
\begin{aligned}
\left\|f_{n}\right\|_{\infty} & =\sup \left\{f_{n}(z): z \in \bar{D}\right\} \\
& =\sup \left\{\left\|A(z)^{2^{n}}\right\|^{1 / 2^{n}}: z \in \bar{D}\right\}=\left\|A^{2^{n}}\right\|_{\infty}^{1 / 2^{n}}=\left\|M_{A}^{2^{n}}\right\|^{1 / 2^{n}},
\end{aligned}
$$

we have

as required.

$$
r=\lim _{n \rightarrow \infty}\left\|f_{n}\right\|_{\infty}=\lim _{n \rightarrow \infty}\left\|M_{A}^{2^{n}}\right\|^{1 / 2^{n}}=\left\|M_{A}\right\|_{s p}
$$

We next have the following result:

Proposition 3. If $\mathscr{E}$ is finite dimensional, and $A: D \rightarrow B(\mathscr{E})$ is a bounded analytic function, then

$$
\rho_{S}\left(M_{A}\right)=\left\|M_{A}\right\|_{\mathrm{sp}}=\sup \left\{\|A(z)\|_{\mathrm{sp}}: z \in D\right\} .
$$

Proof. First note that $M \mapsto\|M\|_{\text {sp }}$ is a continuous map for $M \in B(\mathscr{E})$. Moreover, we have that

$$
\left\|M^{2 n}\right\|^{1 / 2^{n}} \downarrow\|M\|_{\mathrm{sp}} \quad \forall M \in B(\mathscr{E}) .
$$

By Dini's theorem, we have $\left\|M^{2^{n}}\right\|^{1 / 2^{n}} \rightarrow\|M\|_{\mathrm{sp}}$ uniformly for $M$ in any bounded subset of $B(\mathscr{E})$. So if $A(z) \in H^{\infty}\left(B\left(\mathbf{C}^{N}\right)\right)$, we have (using the uniform convergence property just established)

$$
\begin{aligned}
\left\|M_{A}\right\|_{\mathrm{sp}} & =\lim _{n \rightarrow \infty}\left\|M_{A}^{2^{n}}\right\|^{1 / 2^{n}}=\lim _{n \rightarrow \infty} \sup _{z \in D}\left\|A(z)^{2^{n}}\right\|^{1 / 2^{n}} \\
& =\sup _{z \in D} \lim _{n \rightarrow \infty}\left\|A(z)^{2^{n}}\right\|^{1 / 2^{n}}=\sup _{z \in D}\|A(z)\|_{\mathrm{sp}},
\end{aligned}
$$

which completes the proof of the proposition. 
Remark 3. It does not seem possible to significantly weaken the hypotheses of Propositions 2 and 3. Indeed, one can show that if the analytic function $A: D \rightarrow B(\mathscr{E})$ fails to be continuous on $\bar{D}$, and if $\mathscr{E}$ is infinite dimensional, then we may have

$$
\sup _{z \in D}\|A(z)\|_{\mathrm{sp}}<\left\|M_{A}\right\|_{\mathrm{sp}}
$$

(See Example 7 in $\S 7$.

We will use these results in our proof of the spectral commutant lifting theorem in the next section.

\section{Spectral commutant lifting theorem}

In this section, we will prove our main result, namely a spectral analogue of the commutant lifting theorem [24]. This will be applied to the spectral version of classical matricial Nevanlinna-Pick interpolation below.

First we need to recall some general facts about Banach algebras. More precisely, let $\mathscr{A}$ be a complex Banach algebra and let $x \in \mathscr{A}$ be an invertible element whose spectrum does not separate 0 from $\infty$. Then, as is well known, $x=\exp (y)$ for some $y \in \mathscr{A}$. In particular, if $\mathscr{A}$ is finite dimensional, then $\sigma(x)$ is finite for every $x \in \mathscr{A}$, and thus every invertible element $x$ is an exponential.

We thus have the following elementary well-known result which we will use repeatedly throughout this paper:

Lemma 1. Let $\mathscr{H}$ be a finite-dimensional Hilbert space, and let $T \in B(\mathscr{H})$. Then every invertible operator $X \in\{T\}^{\prime}$ can be written as $X=\exp (Y)$ for some $Y \in\{T\}^{\prime}$.

Proof. Apply the preceding remarks to the finite-dimensional algebra $\{T\}^{\prime}$.

We are now ready to state the main result of this section:

Theorem 3 (Spectral commutant lifting theorem). Let $T \in B(\mathscr{H})$ be a contraction with minimal isometric dilation $U \in B(\mathscr{K})$. Let $A \in\{T\}^{\prime}$ be fixed, and set

$$
\operatorname{Dil}(A):=\left\{B \in\{U\}^{\prime}: P_{\mathscr{H}} B=A P_{\mathscr{H}}\right\},
$$

where $P_{\mathscr{H}}: \mathscr{K} \rightarrow \mathscr{H}$ denotes orthogonal projection.

(i) If $\mathscr{K} \ominus \mathscr{H}$ is hyperinvariant for $U$, then

$$
\rho_{T}(A) \leq \inf \left\{\rho_{U}(B): B \in \operatorname{Dil}(A)\right\} .
$$

(ii) If $\mathscr{H}$ is finite dimensional, then

$$
\rho_{T}(A) \geq \inf \left\{\rho_{U}(B): B \in \operatorname{Dil}(A)\right\} .
$$

(iii) If $\mathscr{H}$ is finite dimensional, and $\mathscr{K} \ominus \mathscr{H}$ is hyperinvariant for $U$, then

$$
\rho_{T}(A)=\inf \left\{\|B\|_{\mathrm{sp}}: B \in \operatorname{Dil}(A)\right\} .
$$


Proof. First recall that a subspace $\mathscr{H}^{\prime} \subset \mathscr{K}$ is hyperinvariant for $U$ if it is invariant for every operator in the commutant $\{U\}^{\prime}$ of $U$. Part (iii) is clearly a consequence of (i), (ii), and Proposition 1 . Assume next that $\mathscr{K} \ominus$ $\mathscr{H}$ is hyperinvariant for $U$. Then in this case the map $M \mapsto P_{\mathscr{H}} M \mid \mathscr{H}$ is a homomorphism of $\{U\}^{\prime}$ onto $\{T\}^{\prime}$. Hence, for every $B \in \operatorname{Dil}(A)$, we have

$$
\begin{aligned}
\rho_{U}(B) & =\inf \left\{\left\|M B M^{-1}\right\|: M \in\{U\}^{\prime} \text { invertible }\right\} \\
& \geq \inf \left\{\left\|P_{\mathscr{L}} M B M^{-1} \mid \mathscr{H}\right\|: M \in\{U\}^{\prime} \text { invertible }\right\} \\
& =\inf \left\{\left\|\left(P_{\mathscr{L}} M \mid \mathscr{H}\right) A\left(P_{\mathscr{L}} M \mid \mathscr{H}\right)^{-1}\right\|: M \in\{U\}^{\prime} \text { invertible }\right\} \\
& \geq \inf \left\{\left\|N A N^{-1}\right\|: N \in\{T\}^{\prime} \text { invertible }\right\}=\rho_{T}(A),
\end{aligned}
$$

from which we get (i).

Assume now that $\mathscr{H}$ is finite dimensional. In this case, we claim that every invertible operator $N \in\{T\}^{\prime}$ has the form $P_{\mathscr{H}} M \mid \mathscr{H}$, where $P_{\mathscr{H}} M=N P_{\mathscr{H}}$, $M \in\{U\}^{\prime}$, and $M$ is invertible. Indeed, by Lemma 1 we have that $N=\exp (Y)$ for some $Y \in\{T\}^{\prime}$. If $Z$ is any commuting dilation of $Y$, then $M:=\exp (Z)$ is an invertible commuting dilation of $N$. Now let $\varepsilon>0$, and choose $N \in\{T\}^{\prime}$ such that

$$
\left\|N A N^{-1}\right\|<\rho_{T}(A)+\varepsilon .
$$

Let $M$ be an invertible commuting dilation of $N$, and let $C$ be a commuting dilation of $N A N^{-1}$ such that

$$
\|C\|=\left\|N A N^{-1}\right\| .
$$

Then $B:=M^{-1} C M$ is a commuting dilation of $A$, and

$$
\rho_{U}(B) \leq\left\|M B M^{-1}\right\|=\|C\|<\rho_{T}(A)+\varepsilon .
$$

Since $\varepsilon$ is arbitrary, we thus have that (ii) and the theorem are proven.

Remark 4. The most useful part of Theorem 3 is (iii), and so we would like to discuss its range of applicability. First notice that for $T$ completely nonunitary (i.e., $T$ has no nonzero reducing subspaces on which it is unitary; see [25]), $U$ must be a shift of finite multiplicity. In this case, up to unitary equivalence, we may assume that $U=S$ is the canonical shift on $H^{2}(\mathscr{E})=H^{2} \otimes \mathscr{E}$, where $\mathscr{E}$ is a finite-dimensional (complex) Hilbert space. The hyperinvariant subspaces of $H^{2}(\mathscr{E})$ have the form $m H^{2}(\mathscr{E})$ with $m \in H^{\infty}$ inner (see [5, 25]), and the space $H^{2}(\mathscr{E}) \ominus m H^{2}(\mathscr{E})$ is finite dimensional if and only if $m$ is a finite Blaschke product. Thus the operators $T$ to which (iii) applies have the form $S(m) \otimes I_{\mathscr{E}}$, with $\mathscr{E}$ a finite-dimensional Hilbert space.

Finally, for $T$ as in part (iii) of Theorem 3 with minimal isometric dilation $U$ a shift, we will say that a commuting dilation $B$ of $A \in\{T\}^{\prime}$ is rational if it is defined by multiplication by a rational (matrix-valued) function.

Lemma 2. Assume that the hypotheses of part (iii) of Theorem 3 hold, and that the minimal isometric dilation $U$ of $T$ is a shift. Then for every invertible 
$M \in\{T\}^{\prime}$, there exists an invertible commuting dilation $\widehat{M}$ of $M$ such that $\widehat{M}$ is rational.

Proof. As in the proof of Theorem 3, we take $M=e^{L}, L \in\{T\}^{\prime}$, and we dilate $L$ to $\widehat{L} \in \operatorname{Dil}(L)$. Then $\widehat{M}:=e^{\widehat{L}}$ satisfies

$$
\left\|\widehat{M}-\sum_{n=0}^{p} \frac{1}{n !} \widehat{L}^{n}\right\|:=\eta(p) \rightarrow 0 \quad \text { for } p \rightarrow \infty .
$$

Notice that we can always choose $\widehat{L}$ to be rational, e.g., the maximum entropy solution [11]. On the other hand for every $p \geq 0$,

$$
\widehat{M}_{p}:=\sum_{n=0}^{p} \frac{1}{n !} \widehat{L}^{n}
$$

is rational, and hence so is $\widehat{M}_{p}^{-1}$. Moreover, for $p$ sufficiently large (namely, such that $\left.\eta(p)<\left\|\widehat{M}^{-1}\right\|^{-1}\right)$, we have

$$
\left\|\widehat{M}-\widehat{M}_{p}\right\| \leq \eta(p)
$$

and

$$
\left\|\widehat{M}_{p}^{-1}\right\| \leq\left\|\widehat{M}^{-1}\right\| \frac{\eta(p)}{1-\left\|\widehat{M}^{-1}\right\| \eta(p)} .
$$

So if $\eta(p)<1 / 2\left\|\widehat{M}^{-1}\right\|$ we have

$$
X:=P_{\mathscr{P}}\left(I-\widehat{M}_{p}^{-1} \widehat{M}\right) \mid \mathscr{H}
$$

satisfies $\|X\|<1$. Now choose an exact (norm-preserving) maximum entropy dilation $\widehat{X}$ of $X$ (so that $\widehat{X}$ is rational), and define

$$
\widehat{N}:=\widehat{M}_{p}(I-\widehat{X}) \text {. }
$$

Then $\widehat{N}$ is invertible, and

$$
P_{\mathscr{H}} \widehat{N}\left|\widehat{\mathscr{H}}=P_{\mathscr{H}} \widehat{M}_{p} P_{\mathscr{H}} \widehat{M}_{p}^{-1} \widehat{M}\right| \mathscr{H}=P_{\mathscr{H}} \widehat{M} \mid \mathscr{H}=M,
$$

giving the desired result.

We now have the following key corollary to Theorem 3 .

Corollary 1. For $T$ completely nonunitary, and under the hypotheses of part (iii) of Theorem 3, we have

$$
\rho_{T}(A)=\inf \left\{\left\|M_{B}\right\|_{\mathrm{sp}}: M_{B} \text { is a commuting dilation of } A,\right.
$$

$$
B(z) \text { is rational\}. }
$$

Proof. Denote by $\mu_{0}$ the infimum of the right-hand side of equality (1) above. By Theorem 3, we have that $\rho_{T}(A) \leq \mu_{0}$. Suppose now to the contrary that $\rho_{T}(A)<\mu_{0}$. Then there exists $M \in\{T\}^{\prime}, M$ invertible, such that

$$
\left\|M A M^{-1}\right\|<\mu_{0} .
$$


Now by Lemma 2, we can find an invertible rational commuting dilation $\widehat{M}$ of $M$. Let $B$ be the exact maximum entropy commuting dilation of $M A M^{-1}$. Then $B$ is rational. If we now set

$$
\widehat{B}:=\widehat{M}^{-1} B \widehat{M},
$$

we have that $\widehat{B}$ is rational, from which we can draw the required contradiction to complete the proof of the corollary.

Remarks 5. (i) Corollary 1 means that as in the classical commutant lifting theorem, for $T=S(m) \otimes I_{\mathscr{E}}$ on a finite-dimensional space, we can always find rational commuting dilations arbitrarily close to optimal for the spectral commutant lifting theorem.

(ii) It is possible to extend the spectral commutant lifting theorem to more general contractions $T$ which would allow us to derive the spectral analogues of certain tangential interpolation results as in [12]. Namely, for $T$ a contraction on $\mathscr{H}$, let $U: \mathscr{K} \rightarrow \mathscr{K}$ denote an isometric dilation of $T$. We let $\mathscr{Z}$ be the set of all operators $X: \mathscr{H} \rightarrow \mathscr{H}_{1} \subset \mathscr{K}$ such that $X$ is invertible, $U$ is an isometric dilation of $T_{1}:=X T X^{-1}$, and $X$ has an invertible intertwining dilation. For $A \in\{T\}^{\prime}$, we set

$$
\rho_{T, U}(A):=\inf \left\{\left\|X A X^{-1}\right\|: X \in \mathscr{X}\right\} .
$$

Now for $U$ the minimal isometric dilation of $T$, and for $\mathscr{K} \ominus \mathscr{H}$ hyperinvariant for $U$, we can show $\rho_{T}(A)=\rho_{T, U}(A)$. Moreover, one could prove using the above methods a spectral commutant lifting theorem relative to $\rho_{T, U}(A)$.

The only problem at this point, however, is that we do not know how to explicitly characterize the set $\mathscr{X}$, except using an indirect method like that given in [6]. Because of the obvious relevance of such a description to a more general spectral commutant lifting theorem, we plan to return to this topic in a future paper.

\section{SIMILARITY ORBITS}

In this section we would like to make some general remarks on the properties of $\rho_{T}(A)$ under the action of a certain algebraic group. Namely, following the notation of Theorem 3(iii) for $T=S(m) \otimes I_{\mathscr{E}}$ with $m$ a finite Blaschke product and $\mathscr{E}$ finite-dimensional (see Remark 4), let

$$
\mathscr{G}_{T}:=\left\{M \in\{T\}^{\prime}: M \text { invertible }\right\} .
$$

Set

$$
\mathscr{S}_{T}(A):=\left\{M A M^{-1}: M \in \mathscr{G}_{T}\right\},
$$

the similarity orbit of $A$ with respect to the commutant of $T$. It is obvious that

$$
\rho_{T}(B)=\rho_{T}(A) \quad \forall B \in \mathscr{S}_{T}(A)
$$


We now have

Proposition 4. If $B \in \overline{\mathscr{S}_{T}(A)}$, then $\rho_{T}(B) \geq \rho_{T}(A)$.

Proof. Let $B \in \overline{\mathscr{S}_{T}(A)}$. For $\varepsilon>0$, there exists $M_{\varepsilon} \in \mathscr{G}_{T}$ such that $\| M_{\varepsilon} A M_{\varepsilon}^{-1}$ $-B \|<\varepsilon$. Let $\delta>0$ be fixed. Then there exists $M \in \mathscr{G}_{T}$ satisfying $\left\|M B M^{-1}\right\|<\rho_{T}(B)+\delta$. Consequently,

$$
\begin{aligned}
\left\|M M_{\varepsilon} A\left(M M_{\varepsilon}\right)^{-1}\right\| & \leq\left\|M B M^{-1}\right\|+\left\|M\left(M_{\varepsilon} A M_{\varepsilon}^{-1}-B\right) M^{-1}\right\| \\
& <\rho_{T}(B)+\delta+\|M\|\left\|M^{-1}\right\| \varepsilon .
\end{aligned}
$$

To finish the proof, we first let $\varepsilon \downarrow 0$, and then $\delta \downarrow 0$.

Remark 6. In fact we may have $\rho_{T}(B)>\rho_{T}(A)$; see Example 1 in $\S 7$.

Proposition 5. If there exists $M_{0} \in \mathscr{G}_{T}$ such that

$$
\rho_{T}(A)=\left\|M_{0} A M_{0}^{-1}\right\|,
$$

then there exists $B_{0}$, a commuting dilation of $A$, which is rational, and such that

$$
\left\|B_{0}\right\|_{\mathrm{sp}}=\rho_{T}(A)
$$

Proof. Similar proof as in Corollary 1.

We would now like to study the orbit structure for diagonalizable $A$.

Proposition 6. Assume the notation given above. If $A \in\{T\}^{\prime}$ is diagonalizable, then $\mathscr{S}_{T}(A)=\overline{\mathscr{S}_{T}(A)}$.

Proof. Throughout this proof, we will identify our linear operators (defined on finite-dimensional vector spaces) with their matrix representations. Without loss of generality, we may assume that $T$ has only one eigenvalue (i.e., that $m$ has only one zero up to multiplicity). Thus $T$ is isomorphic to a direct sum of Jordan blocks all with the same eigenvalues. Let $\mathscr{V}_{1}, \ldots, \mathscr{V}_{p}$ denote eigenspaces of the diagonalizable operator $A \in\{T\}^{\prime}$. Note that $T\left(\mathscr{V}_{j}\right) \subset \mathscr{V}_{j}, j=1, \ldots, p$, and so we can write

$$
T \mid \mathscr{V}_{j} \cong \bigoplus_{k} \mathscr{J}_{j k}
$$

for appropriate (uniquely defined up to permutation) Jordan blocks, and hence

$$
T \cong \bigoplus_{j} \bigoplus_{k} \mathscr{J}_{j k} .
$$

Consequently, we see that there exists $M \in\{T\}^{\prime}$ invertible, such that $M A=$ $D_{A} M$ for $D_{A}$ a diagonal matrix (which obviously has the same eigenstructure as $A$ ). Notice that the diagonal entries of $D_{A}$ corresponding to any Jordan block of $T$ are identical.

Next it is well known [26] that if

$$
\mathscr{S}(A):=\left\{X A X^{-1}: X \text { invertible }\right\},
$$


then $\mathscr{S}(A)$ is closed. Let $A^{\prime} \in \overline{\mathscr{S}_{T}(A)}$, and let

$$
M_{j} A M_{j}^{-1} \rightarrow A^{\prime}, \quad M_{j} \in\{T\}^{\prime} \text {. }
$$

Then $A^{\prime} \in \overline{\mathscr{S}(A)}=\mathscr{S}(A)$, and moreover it is easy to see that $A^{\prime} \in\{T\}^{\prime}$. Consequently, by the argument given in the first paragraph of this proof, there exists $\widetilde{M} \in\{T\}^{\prime}, \widetilde{M}$ invertible, such that

$$
\widetilde{M} A^{\prime}=D_{A^{\prime}} \widetilde{M}
$$

where $D_{A^{\prime}}$ is diagonal (with the same eigenstructures as $A^{\prime}$ ). Since all the Jordan blocks of $T$ have the same size, we see therefore that there exists a permutation matrix $P$ such that $P M A^{\prime}=A P M$, which implies the desired result.

Remark 7. The referee of this paper kindly pointed out to us an alternative proof to Proposition 6 which we would like to state here. The following neat argument of the referee actually works for $T$ an arbitrary operator on a finitedimensional space and not just $T=S(m) \otimes I_{\mathscr{C}}$.

The proof begins by noting as above that for $A^{\prime} \in \overline{\mathscr{S}_{T}(A)}, A^{\prime}$ is similar to $A$ and $A \in\{T\}^{\prime}$. Now let $P_{1}, \ldots, P_{r}$ be the spectral projections onto the eigenspaces of $A^{\prime}$ given by the Riesz-Dunford functional calculus. Clearly $P_{j} \in\{T\}^{\prime}$ for $1 \leq j \leq r$. Next for $A^{\prime \prime} \in \mathscr{S}_{T}(A)$, let $Q_{1}, \ldots, Q_{r}$ be the corresponding spectral projections. Since we can choose $A^{\prime \prime}$ so that $\left\|A^{\prime}-A^{\prime \prime}\right\|$ is arbitrarily small, we can choose $A^{\prime \prime}$ so that

$$
\left\|\left(A^{\prime}-z I\right)^{-1}-\left(A^{\prime \prime}-z I\right)^{-1}\right\|
$$

is as small as desired uniformly on any compact subset of the plane not containing the eigenvalues of $A$. Thus, we can find $A^{\prime \prime} \in \mathscr{S}_{T}(A)$ such that $\left\|P_{j}-Q_{j}\right\|<1 \forall j$. Hence the operator $P_{j} Q_{j}$ gives a one-to-one map of the $j$ th eigenspace of $A^{\prime \prime}$ onto the $j$ th eigenspace of $A^{\prime}$. We therefore can conclude that the operator

$$
M:=\sum_{j=1}^{n} P_{j} Q_{j}
$$

is invertible. Since clearly $M \in\{T\}^{\prime}$, and interwines $A^{\prime \prime}$ with $A^{\prime}$, we are done.

Corollary 2. If $A$ is diagonalizable, then there exists $B_{0}$, a commuting dilation of $A$, which is rational and satisfies equation (3).

Proof. This follows immediately from Propositions 5 and 6.

\section{Spectral NevanlinNa-Pick theory}

In this section, we apply the above theory to a spectral version of the Nevanlinna-Pick interpolation problem. In order to do this, we first put the Nevanlinna-Pick theory into the commutant lifting framework [22, 24, 25]. 
First let us recall the general problem of Nevanlinna-Pick in the matrix case. See also the closely related discussion in $\S 2$ in the scalar case.

Let $\mathscr{E}$ be a finite-dimensional vector space, let $z_{1}, \ldots, z_{n} \in D$ be mutually distinct, and let $F_{1}, \ldots, F_{n} \in B(\mathscr{E})$. Then we want necessary and sufficient conditions for the existence of an analytic function $F: D \rightarrow B(\mathscr{E})$ with $\|F\|_{\infty} \leq 1$ such that $F\left(z_{j}\right)=F_{j}$ for $j=1, \ldots, n$. Define

$$
m(z):=\prod_{j=1}^{n} \frac{z-z_{j}}{1-\bar{z}_{j} z}
$$

and

$$
f_{j}:=\left(\prod_{k \neq j} \frac{z-z_{k}}{1-\bar{z}_{k} z}\right) \frac{1}{1-\bar{z}_{j} z} .
$$

We set $T:=S(m) \otimes I_{\mathscr{E}}$, and note that

$$
\mathscr{H}=f_{1} \otimes \mathscr{E}+f_{2} \otimes \mathscr{E}+\cdots+f_{n} \otimes \mathscr{E} \text {. }
$$

This sum is direct but not orthogonal.

For the given interpolation data above, we define $A: \mathscr{H} \rightarrow \mathscr{H}$ by linearity and by

$$
A\left(f_{j} \otimes \zeta\right)=f_{j} \otimes F_{j} \zeta
$$

for all $\zeta \in \mathscr{E}, j=1,2, \ldots, n$. Note that $T\left(f_{j} \otimes \zeta\right)=z_{j} f_{j} \otimes \zeta$ for all $\zeta \in \mathscr{E}, j=1,2, \ldots, n$. Thus $A \in\{T\}^{\prime}$.

As in the proof of Theorem 2, $F$ satisfies the interpolation conditions

$$
F\left(z_{j}\right)=F_{j} \quad \forall j=1, \ldots, n
$$

if and only if

$$
P_{\mathscr{H}} M_{F}=A P_{\mathscr{H}}
$$

Thus, the commutant lifting theorem implies that there exists an $F$ satisfying (4) and $\|F\|_{\infty} \leq 1$, if and only if $\|A\| \leq 1$. We will now show that the spectral Nevanlinna-Pick problem can be given a similar solution, based on the spectral commutant lifting theorem.

Theorem 4. The following properties are equivalent:

(i) There exists a bounded analytic function $F: D \rightarrow B(\mathscr{E})$ satisfying

$$
F\left(z_{j}\right)=F_{j} \quad(1 \leq j \leq n)
$$

and such that $\left\|M_{F}\right\|_{\mathrm{sp}}<1$.

(ii) There exists a bounded rational function $F: \bar{D} \rightarrow B(\mathscr{E})$, satisfying the interpolation conditions (5) and such that $\|F(z)\|_{\mathrm{sp}}<1$ for all $z \in \bar{D}$.

(iii) $\rho_{T}(A)<1$.

Proof. We have that (iii) $\Leftrightarrow$ (i) by our above discussion and Theorem 3 . Finally (ii) $\Leftrightarrow$ (i) from Corollary 1 . 
Corollary 3. If all the matrices $F_{1}, \ldots, F_{n}$ are diagonalizable, then there exists a bounded rational function $F: \bar{D} \rightarrow \mathscr{E}$ satisfying (5) above, and such that

$$
\rho_{T}(A)=\max _{z \in \bar{D}}\|F(z)\|_{\mathrm{sp}}
$$

Proof. The proof follows at once from Corollary 2 and the above discussion.

Remark 8. Note that the condition that $F_{1}, \ldots, F_{n}$ are diagonalizable is a generic condition on the space $B(\mathscr{E})$.

In order to state our next result we will need to set up some more notation. First note (using the notation above) that if $M \in\{T\}^{\prime}$, then necessarily

$$
M\left(f_{j} \otimes \zeta\right)=f_{j} \otimes M_{j} \zeta
$$

for all $\zeta \in \mathscr{E}$ and where $M_{j} \in B(\mathscr{E})$ for $j=1, \ldots ; n$. $M$ is invertible if and only if each $M_{j}(1 \leq j \leq n)$ is invertible.

We denote the operator $A$ associated with the matrices $F_{1}, \ldots, F_{n}$ by $A\left(F_{1}, \ldots, F_{n}\right)$. Then

$$
M A M^{-1}=A\left(M_{1} F_{1} M_{1}^{-1}, \ldots, M_{n} F_{n} M_{n}^{-1}\right) .
$$

Clearly

$$
\begin{aligned}
& \rho_{T}\left(A\left(F_{1}, \ldots, F_{n}\right)\right) \\
& =\inf \left\{\left\|A\left(M_{1} F_{1} M_{1}^{-1}, \ldots, M_{n} F_{n} M_{n}^{-1}\right)\right\|: M_{j} \in B(\mathscr{E})\right. \\
& \left.1 \leq j \leq n, M_{j} \text { invertible }\right\}
\end{aligned}
$$

and

$$
\rho_{T}\left(A\left(F_{1}, \ldots, F_{n}\right)\right)=\rho_{T}\left(A\left(J_{1}, \ldots, J_{n}\right)\right),
$$

where $J_{j}$ is the Jordan form of the matrix $F_{j}$ for $1 \leq j \leq n$.

Corollary 4. If $D_{1}, \ldots, D_{n}$ denote the diagonalizable parts of $F_{1}, \ldots, F_{n}$ respectively, then

$$
\rho_{T}\left(A\left(D_{1}, \ldots, D_{n}\right)\right) \geq \rho_{T}\left(A\left(F_{1}, \ldots, F_{n}\right)\right) .
$$

Proof. The proof follows at once from Proposition 4, and the above discussion.

Remark 9. Corollary 4 implies that if we can solve the spectral Nevanlinna-Pick problem for the diagonal parts of the Jordan canonical forms of the interpolation data $F_{1}, \ldots, F_{n}$, then we can solve the problem for the original data. Thus this gives a sufficient condition for the solution. (See $\S 7$ for examples which show that the condition is certainly not necessary.)

We can now state:

Corollary 5. Suppose that $F_{1}, \ldots, F_{n}$ are diagonalizable. Then the following properties are equivalent:

(i) There exists a bounded rational function $F: D \rightarrow B(\mathscr{E})$ satisfying the interpolation conditions (5) and such that $\max _{z \in \bar{D}}\|F(z)\|_{\mathrm{sp}} \leq 1$. 
(ii) There exists $B$, a commuting dilation of $A=A\left(F_{1}, \ldots, F_{n}\right)$, such that $\|B\|_{\mathrm{sp}} \leq 1$.

(iii) $\rho_{T}(A) \leq 1$.

Proof. Immediate from Theorem 3, Corollary 3, and our above discussion.

Remarks 10. (i) We should note that in Corollary 5 (i) and (ii) are equivalent, and (i) implies (iii) without the hypothesis of genericity that the interpolation data $F_{1}, \ldots, F_{n}$ are diagonalizable. (This follows from Theorem 3.) However, at this point we do not know if (iii) implies (i) in this generality .

(ii) In Examples 5 and 6 in $\S 7$, we show that an optimal solution may not be diagonalizable even when we start with diagonalizable interpolation data. Moreover, in Example 3 we show that there may not be a common bound on the norms of the optimal solutions.

(iii) We should note that there exist computer algorithms for checking the condition that $\rho_{T}(A) \leq 1$, and for constructing optimal $M$. Indeed such techniques based on gradient search procedures are described in [10,17]. Hence we can actually employ the above methods to algorithmically solve the spectral Nevannlinna-Pick problem in much the same way that the classical problem is solved. This is important since much of the original motivation for considering such questions came from control theoretic and engineering questions. (See [7, $10,26]$ and the references therein.)

\section{EXAMPLES AND COUNTEREXAMPLES}

In this section, we discuss a number of illuminating examples to clarify some of the results on spectral dilation theory. We should explain first some of the motivation behind these examples.

As we noted in the Introduction, one may deduce many of the classical interpolation results using the hyperbolic structure of the unit disc. This even extends to the matrix case, since the ball of complex square matrices of radius 1 is again hyperbolic [18]. This type of hyperbolic geometric approach and its relevance to modern interpolation theory has greatly influenced the work of Ball-Helton [4].

Now one may attempt to play the same game in the spectral case. Namely, we are considering analytic matrix-valued interpolating functions from the unit disc to the space of square complex matrices (of fixed size $N \times N$ ) of spectral radius bounded by 1 . This space is not hyperbolic since the Kobayashi metric [18] degenerates (that is, is only a pseudo-metric). (To see this, just consider the line of matrices with spectral radius $<1$ given by

$$
\left.\left[\begin{array}{cc}
1 / 2 & t \\
0 & 1 / 2
\end{array}\right] \text { for } t \in \mathbf{C} .\right)
$$

In fact, it is easy to prove that in the Kobayashi pseudo-metric the distance between a matrix and the diagonal part of its Jordan canonical form is 0 . 
Based on the classical hyperbolic approach to interpolation, this would lead one to conjecture that one could solve the Nevanlinna-Pick spectral interpolation problem for given data $F_{1}, \ldots, F_{n}$ if and only if it could be solved for the diagonal parts of the Jordan canonical forms of the data. We have seen in Remark 4 that one direction $(\Leftarrow)$ holds. One of the points of the discussion below will be to show that the $(\Rightarrow)$ direction breaks down completely. This will be illustrated by elucidating the structure of $\overline{\mathscr{S}_{T}(A)}$.

Hence in the spectral case, there seem to be a number of truly new interpolation phenomena occurring, for which the classical intuition no longer applies.

Example 1. Our first example shows that $\rho_{T}$ need not be constant on $\overline{\mathscr{S}_{T}(A)}$, even though as we have seen above, it is constant on $\mathscr{S}_{T}(A)$.

Let $z_{1}=0, z_{2}=z_{0} \in D \backslash\{0\}$, and consider

$$
F_{1}:=\left[\begin{array}{ll}
0 & 0 \\
1 & 0
\end{array}\right], \quad F_{2}:=\left[\begin{array}{cc}
0 & z_{0} \\
1 & 0
\end{array}\right] .
$$

Obviously, the diagonalizable parts are

$$
D_{1}:=\left[\begin{array}{ll}
0 & 0 \\
0 & 0
\end{array}\right], \quad D_{2}:=F_{2} .
$$

Moreover, $F(z):=\left[\begin{array}{ll}0 & z \\ 1 & 0\end{array}\right]$ satisfies $F\left(z_{j}\right)=F_{j}(j=1,2)$ and

$$
\|F(z)\|_{\mathrm{sp}} \leq 1 \quad \forall z \in \bar{D} .
$$

Consequently,

$$
\rho_{T}\left(A\left(F_{1}, F_{2}\right)\right) \leq 1 \text {. }
$$

Suppose that $\rho_{T}\left(A\left(F_{1}, F_{2}\right)\right)=\rho_{T}\left(A\left(D_{1}, D_{2}\right)\right)$. Then there would exist

$$
G(z):=z\left[\begin{array}{ll}
f(z) & g(z) \\
h(z) & k(z)
\end{array}\right]
$$

rational satisfying

$$
f\left(z_{0}\right)=0=k\left(z_{0}\right), \quad g\left(z_{0}\right)=1, \quad h\left(z_{0}\right)=1 / z_{0} .
$$

Now

$$
d(z):=\operatorname{det} G(z)=z^{2}(f(z) k(z)-g(z) h(z)):=z^{2} d_{1}(z)
$$

where $\left|d_{1}(z)\right| \leq 1$ on $\partial D$, and thus also on $D$. But $\left|d_{1}\left(z_{0}\right)\right|=\left|1 / z_{0}\right|$, a contradiction.

Notice moreover that for this example we have that

$$
\sqrt{\left|z_{0}\right|}=\|A\|_{\mathrm{sp}}<\rho_{T}(A)=1 .
$$

Example 2. The same interpolating function as in Example 1 can be used to show that the data $F_{j}$ can be diagonalizable, and yet none of the exact interpolating functions $F(z)$, i.e., those satisfying

$$
\sup _{z \in D}\|F(z)\|_{\mathrm{sp}}=\rho_{T}\left(A\left(F_{1}, \ldots, F_{n}\right)\right),
$$


are diagonalizable. Indeed let $z_{1}, z_{2} \in D$ be distinct points which are nonzero, and set

$$
F_{1}:=\left[\begin{array}{cc}
0 & z_{1} \\
1 & 0
\end{array}\right], \quad F_{2}:=\left[\begin{array}{cc}
0 & z_{2} \\
1 & 0
\end{array}\right] .
$$

Since $F(z)=\left[\begin{array}{ll}0 & z \\ 1 & 0\end{array}\right]$ is an interpolating function with $\sup _{z \in D}\|F(z)\|_{\mathrm{sp}}=1$, we have that

$$
\rho_{T}\left(A\left(F_{1}, F_{2}\right)\right) \leq 1 .
$$

Let now $\widetilde{F}$ be any other bounded solution satisfying

$$
\sup _{z \in D}\|\widetilde{F}(z)\|_{\mathrm{sp}} \leq 1 \text {. }
$$

Then

$$
|\operatorname{det} \widetilde{F}(z)| \leq 1 \quad \forall z \in D
$$

and

$$
\operatorname{det} \widetilde{F}\left(z_{1}\right)=-z_{1}, \quad \operatorname{det} \widetilde{F}\left(z_{2}\right)=-z_{2},
$$

and therefore, by virtue of the uniqueness in the Schur problem,

$$
\operatorname{det} \widetilde{F}(z)=-z \quad \forall z \in D .
$$

Set $\theta(z):=\frac{1}{2}$ trace $\widetilde{F}(z)$. Then we have

$$
\left|\theta(z) \pm \sqrt{\theta(z)^{2}+z}\right| \leq 1 \quad \forall z \in D
$$

and so by the parallelogram law

$$
2|\theta(z)|^{2}+2\left|\theta(z)^{2}+z\right| \leq 2 \quad \forall z \in D,
$$

whence

$$
|\theta(\zeta)|^{2}+\left|\theta(\zeta)^{2}+\zeta\right| \leq 1
$$

for almost every $\zeta \in \partial D$.

Now the triangle inequality shows that

$$
1=|\zeta|=\left|-\theta(\zeta)^{2}+\theta(\zeta)^{2}+\zeta\right| \leq|\theta(\zeta)|^{2}+\left|\theta(\zeta)^{2}+\zeta\right|
$$

whence we deduce that

$$
\theta(\zeta)^{2}=\gamma(\zeta) \zeta
$$

with $\gamma(\zeta) \in[-1,0]$ for almost every $\zeta \in \partial D$. Therefore $\bar{\zeta} \theta(\zeta)^{2} \leq 0$ almost everywhere on $\partial D$. It follows that the bounded harmonic function

$$
\alpha(z):=\theta(0)^{2} \bar{z}+\frac{\theta(z)^{2}-\theta(0)^{2}}{z}
$$

satisfies $\alpha(z) \leq 0$ on $D$, and so in particular is real-valued. Therefore,

$$
\frac{\partial}{\partial z}\left(\frac{\theta(z)^{2}-\theta(0)^{2}}{z}\right)=\frac{\partial}{\partial z} \alpha(z)=\overline{\frac{\partial}{\partial \bar{z}} \alpha(z)}=\overline{\theta(0)^{2}}
$$

so that

$$
\theta(z)^{2}=\theta(0)^{2}+z \overline{\theta(0)}^{2} \quad \forall z \in D
$$


Consequently, if $\theta(z)=\theta(0)+z \psi(z)$, we have

$$
\theta(0)^{2}+2 z \theta(0) \psi(z)+z^{2} \psi(z)^{2}=z \overline{\theta(0)}^{2} \quad \forall z \in D
$$

which means that $\psi(z)=0$ for all $z \in D$.

Thus, we see that $\theta(z)=0$ for all $z \in D$. In particular

$$
\text { trace } \widetilde{F}(0)=0=\operatorname{det} \widetilde{F}(0) .
$$

Now if $\widetilde{F}(z)$ were diagonalizable, this would imply that $\widetilde{F}(z)=z F_{1}(z)$ with $F_{1}$ a bounded analytic $2 \times 2$ matrix-valued function. Therefore, we would have

$$
-z=z^{2} \operatorname{det} F_{1}(z) \quad \forall z \in D
$$

which is clearly absurd. Thus if $\widetilde{F}(z)$ is any interpolating function $\left(\widetilde{F}\left(z_{j}\right)=\right.$ $\left.F_{j}, \quad j=1,2\right)$ satisfying $(9)$, then $\widetilde{F}(0)$ is properly nilpotent (i.e., $\widetilde{F}(0)$ is nilpotent but not the zero matrix).

Example 3. The interpolation data of Example 1 may be slightly modified to prove the existence of exact interpolating functions $F(z)$ (see Example 2), of arbitrarily large norm. Indeed, set $z_{1}=0, z_{2}=z_{0} \neq 0 \quad\left(z_{0} \in D\right)$, and let

$$
F_{1}^{\varepsilon}=\left[\begin{array}{cc}
0 & 0 \\
\varepsilon & 0
\end{array}\right], \quad F_{2}=\left[\begin{array}{cc}
0 & z_{0} \\
1 & 0
\end{array}\right]
$$

for $\varepsilon>0$. Then from the above there exists an interpolating function $F^{\varepsilon}(z)$ with

$$
\sup _{z \in D}\left\|F^{\varepsilon}(z)\right\|_{\mathrm{sp}}=\rho_{T}\left(A\left(F_{1}, F_{2}\right)\right)=1 .
$$

We claim that

$$
\left\|F^{\varepsilon}\right\|_{\infty} \rightarrow \infty \quad \text { as } \varepsilon \rightarrow 0 .
$$

Indeed, suppose not. Then (choosing a subsequence if necessary), we can infer the existence of an analytic function $F^{0}(z)=\lim _{\varepsilon \rightarrow 0} F^{\varepsilon}(z)$ with

$$
F^{0}(0)=\left[\begin{array}{ll}
0 & 0 \\
0 & 0
\end{array}\right], \quad F\left(z_{0}\right)=\left[\begin{array}{cc}
0 & z_{0} \\
1 & 0
\end{array}\right],
$$

and such that $\left\|M_{F^{0}}\right\|_{\text {sp }}=1$. A similar argument to that given in Example 2 immediately leads to the required contradiction.

We should also remark here that whenever we have $A^{\prime} \in \overline{\mathscr{S}}_{T}(A) \backslash \mathscr{S}_{T}(A)$ and $\rho_{T}\left(A^{\prime}\right)>\rho_{T}(A)$, we have an identical phenomenon of arbitrarily increasing norm to that described above.

Example 4. It is easy to write down an example to show that the infimum defining $\rho_{T}(A)$ is not necessarily a minimum, that, is, we may have

$$
\rho_{T}(A)<\left\|A^{\prime}\right\| \quad \forall A^{\prime} \in \mathscr{S}_{T}(A) .
$$

More precisely, let

$$
T:=\left[\begin{array}{ll}
0 & 0 \\
0 & 0
\end{array}\right], \quad A:=\left[\begin{array}{ll}
0 & 1 \\
0 & 0
\end{array}\right]
$$


Then $\rho_{T}(A)=\|A\|_{\mathrm{sp}}=0$, but $\left\|M A M^{-1}\right\|>0$ whenever $M \in\{T\}^{\prime}$.

Example 5. Again consider the interpolation problem (5) with data $z_{1}, \ldots, z_{n}$ $\in D$ and $F_{1}, \ldots, F_{n} N \times N$ matrices. This example will show that even if the data $F_{1}, \ldots, F_{n}$ are diagonalizable, $A\left(F_{1}, \ldots, F_{n}\right)$ has distinct eigenvalues, and there is a solution to the interpolation problem $F(z)$ such that

$$
\sup _{z \in D}\|F(z)\|_{\mathrm{sp}}=\sup _{z \in D}\|F(z)\|=\rho_{T}\left(A\left(F_{1}, \ldots, F_{n}\right)\right)
$$

and $F(z)$ is diagonalizable for all $z \in D$, this $F$ cannot be obtained by interpolating the eigenvalues of the data. In other words, even in this case we cannot reduce spectral Nevanlinna-Pick to a series of scalar Nevanlinna-Pick problems.

Indeed, let $z_{1}, z_{2}, z_{3}, z_{4} \in D$ be distinct nonzero points such that $z_{i} \neq$ $z_{j}^{2}, \forall 1 \leq i, j \leq 4$, and set

$$
F_{j}:=\left[\begin{array}{cc}
0 & z_{j} \\
z_{j}^{2} & 0
\end{array}\right] \quad \forall 1 \leq i, j \leq 4
$$

Then

$$
F_{0}(z):=\left[\begin{array}{cc}
0 & z \\
z^{2} & 0
\end{array}\right], \quad z \in D,
$$

is an interpolating solution such that

$$
\sup _{z \in D}\left\|F_{0}(z)\right\|_{\mathrm{sp}}=\sup _{z \in D}\left\|F_{0}(z)\right\|=1 .
$$

Thus

$$
\rho_{T}\left(A\left(F_{1}, F_{2}, F_{3}, F_{4}\right)\right) \leq 1 .
$$

Let now $F$ be any interpolating solution satisfying

$$
\|F(z)\|_{\mathrm{sp}} \leq 1 \quad \forall z \in D .
$$

Then $|\operatorname{det} F(z)| \leq 1$ in $D$ and

$$
\operatorname{det} F\left(z_{j}\right)=-z_{j}^{3}, \quad j=1,2,3,4 \text {. }
$$

Set

$$
m(z):=\prod_{j=1}^{4} \frac{z-z_{j}}{1-\bar{z}_{j} z}
$$

and consider $S(m)^{3}$ on $H(m)$. (Recall from $\S 2$ that $H(m)=H^{2} \ominus m H^{2}$ and $S(m)$ is the compressed shift.) Let $\mathscr{D}_{S(m)}:=\left(I-S(m)^{*} S(m)\right)^{1 / 2} H(m)$. If $0 \neq h_{0} \perp \mathscr{D}_{S(m)} \vee S(m)^{*} \mathscr{D}_{S(m)} \vee S(m)^{* 2} \mathscr{D}_{S(m)}$ (which is of codimension $\geq 1$ ), then

$$
\left\|S(m)^{3} h_{0}\right\|=\left\|h_{0}\right\| \neq 0 .
$$

Hence by the uniqueness in the commutant lifting theorem ( $h_{0}$ is a maximal vector for $S(m)^{3}$; see [22])

$$
\operatorname{det} F(z)=-z^{3} \quad \forall z \in D .
$$


This implies $\sup _{z \in D}\|F(z)\|_{\mathrm{sp}}=1$, and thus

$$
\rho_{T}\left(A\left(F_{1}, F_{2}, F_{3}, F_{4}\right)\right)=1 \text {. }
$$

On the other hand, if we interpolate the eigenvalues $\pm \sqrt{z_{j}^{3}}(1 \leq j \leq 4)$, any solution $\psi$ produces an operator $A_{0}=\psi(S(m))$ such that $A_{0}^{2}=S(m)^{3}$. Since $\left\|S(m)^{3}\right\|=1$, we have that $\left\|A_{0}\right\| \geq 1$. We claim that $\left\|A_{0}\right\|>1$. Indeed, suppose to the contrary that $\left\|A_{0}\right\|=1$. Then the norm of $A_{0}$ would be attained, and therefore $A_{0}=\psi(S(m))$, where $\psi$ is a finite Blaschke product. But in this case we would have

$$
\left\|h_{0}\right\|=\left\|\psi^{2} h_{0}\right\| \geq\left\|P_{H(m)} \psi^{2} h_{0}\right\|=\left\|A_{0}^{2} h_{0}\right\|=\left\|S(m)^{3} h_{0}\right\|=\left\|h_{0}\right\|
$$

and

$$
\left\|h_{0}\right\|=\left\|S(m)^{3} h_{0}\right\|=\left\|P_{H(m)} z^{3} h_{0}\right\| \leq\left\|z^{3} h_{0}\right\|=\left\|h_{0}\right\|
$$

(since $S(m)^{3}$ attains its norm on $h_{0}$ ). Hence

$$
\psi^{2} h_{0}=A_{0}^{2} h_{0}=S(m)^{3} h_{0}=z^{3} h_{0} .
$$

Since $h_{0} \neq 0$, it follows that $\psi(z)^{2}=z^{3} \forall z \in D$, which is obviously impossible. Hence $\left\|A_{0}\right\|>1$ as claimed.

Now since $\left\|A_{0}\right\|>1$ and there are only $2^{4}$ such operators $A_{0}$, we have $\|\psi\|_{\infty}>1$ for any interpolation $\psi$ of the eigenvalues. Hence from (16) for any such interpolating function,

$$
\|\psi\|_{\infty}>\rho_{T}\left(A\left(F_{1}, F_{2}, F_{3}, F_{4}\right)\right),
$$

which completes the example.

Example 6. Let $F_{0}(z)$ be as in Example 5. Notice that $F_{0}$ is an optimal solution to a spectral Nevanlinna-Pick problem which is diagonalizable for all $z \in D$. However $F_{0}(0)=0$ (the zero matrix). It is easy to write down another optimal solution $\widetilde{F}$ to the spectral Nevanlinna-Pick problem with data given by (10) such that $\widetilde{F}(0)$ is nilpotent, but not the zero matrix.

Explicitly, set

$$
\widetilde{F}(z):=\left[\begin{array}{cc}
0 & z^{3} \\
1 & 0
\end{array}\right]
$$

Then clearly $\widetilde{F}(z)$ is not diagonalizable at $z=0$, and $\widetilde{F}$ is similar to

$$
F_{0}(z)=\left[\begin{array}{cc}
0 & z \\
z^{2} & 0
\end{array}\right]
$$

for every $z \neq 0$.

Example 7. Referring to Propositions 2 and 3 in the last example, we will show that if $A$ is not continuous on $\bar{D}$ and $\mathscr{E}$ is infinite dimensional, then we may have

$$
\sup _{z \in D}\|A(z)\|_{\mathrm{sp}}<\left\|M_{A}\right\|_{\mathrm{sp}} .
$$


Indeed, to see this let us denote by $l_{+}^{2}$ the Hilbert space of all square-summable one-sided complex sequences, $\left\{a_{n}\right\}_{n=1}^{\infty}$. Let $e_{n}, n \geq 1$, denote the standard orthonormal basis of $l_{+}^{2}$. Then we define an analytic function $A: D \rightarrow B\left(l_{+}^{2}\right)$ which is given in the basis $e_{n}$ as

$$
\left\langle A(z) e_{j}, e_{i}\right\rangle:= \begin{cases}z^{2^{i-1}}, & j=i+1, \\ 0, & \text { otherwise }\end{cases}
$$

for each $z \in D$. Now it is easy to check that $\left\|A(z)^{n}\right\|=|z|^{n^{2}}$ so that

$$
\left\|A(z)^{n}\right\|^{1 / n}=|z|^{n} \quad \forall z \in D
$$

which implies $\|A(z)\|_{\mathrm{sp}}=0$. On the other hand

$$
\sup _{z \in D}\left\|A(z)^{n}\right\|=1 \quad \forall n \geq 1, \forall z \in D
$$

so that $\left\|M_{A}\right\|_{\mathrm{sp}}=1$.

\section{REFERENCES}

1. V. M. Adamjan, D. Z. Arov, and M. G. Krein, Analytic properties of Schmidt pairs for a Hankel operator and the generalized Shur-Takagi problem, Math. USSR-Sb. 15 (1971), 31-73.

2. __ Infinite Hankel block matrices and related extension problems, Amer. Math. Soc. Transl. 111 (1978), 133-156.

3. L. Ahlfors, Conformal invariants, Topics in Geometric Function Theory, McGraw-Hill, New York, 1973.

4. J. Ball and J. W. Helton, A Beurling-Lax theorem for the Lie group $U(m, n)$ which contains most classical interpolation theory, J. Operator Theory 9 (1983), 107-142.

5. H. Bercovici, Operator theory and arithmetic in $H^{\infty}$, Math. Surveys and Monographs, vol. 26, Amer. Math. Soc., Providence, R.I., 1988.

6. H. Bercovici, C. Foias, and A. Tannenbaum, On skew Toeplitz operators. I, Oper. Theory: Adv. Appl. 29 (1988), 21-43.

7. __ Spectral radius interpolation and robust control, submitted to the 28th CDC, 1989.

8. G. Arsene, Z. Ceausescu, and C. Foias, On intertwining dilations. VII, Lecture Notes in Math., vol. 747, Springer-Verlag, 1979, pp. 24-45.

9. __ On intertwining dilations. VIII, J. Operator Theory 4 (1980), 55-91.

10. J. C. Doyle, Lecture notes in advances in multivariable control, ONR/Honeywell Workshop, Minneapolis, Minn., 1984.

11. H. Dym and I. Gohberg, A maximum entropy principle for contractive interpolants, J. Funct. Anal. 65 (1986), 83-125.

12. I. P. Fedcina, A description of the solutions of the Nevanlinna-Pick tangent problem, Akad. Nauk Armyan. SSR Dokl. 60 (1975), 37-42.

13. C. Foias, Contractive intertwining dilations and waves in layered media, Proc. Internat. Congr. Math., vol. 2, 1978, pp. 605-613.

14. B. A. Francis, $A$ course in $H^{\infty}$ control theory, Lecture Notes in Control and Inform. Sci., vol. 88, Springer-Verlag, 1987.

15. B. A. Francis and A. Tannenbaum, Generalized interpolation in control theory, Math. Intelligencer 10 (1988), 48-53.

16. J. Garnett, Bounded analytic functions, Academic Press, New York, 1981. 
17. J. W. Helton, Optimization in operator theory, analytic function theory and electrical engineering, CBMS Regional Conf. Ser. Math., no. 68, Amer. Math. Soc., Providence, R. I., 1985.

18. S. Kobayashi, Hyperbolic manifolds and holomorphic mappings, Marcel Dekker, New York, 1970.

19. R. Nevanlinna, Über beschrankte Funftionen, die in gegebenen Punkten vorgeschriebene Werte annehmen, Ann. Acad. Sci. Fenn. 13 (1919), 27-43.

20. G. Pick, Über die Beschränkungen analytischer Funktionen, welche durch vorgegebenen Funktionswerte bewiskt sind, Math. Ann. 77 (1916), 7-23.

21. G. C. Rota, On models for linear operators, Comm. Pure Appl. Math. 13 (1960), 468-472.

22. D. Sarason, Generalized interpolation in $H^{\infty}$, Trans. Amer. Math. Soc. 127 (1967), 179 203.

23. B. Sz.-Nagy, Sur les contractions de l'espace de Hilbert, Acta Sci. Math. (Szeged) 15 (1953), 87-92.

24. B. Sz.-Nagy and C. Foias, Dilation des commutants, C.R. Acad. Sci. Paris Sér. A 266 (1968), 493-495.

25. __ Harmonic analysis of operators on Hilbert space, North-Holland, 1970.

26. A. Tannenbaum, Invariance and system theory: algebraic and geometric aspects, Lecture Notes in Math., vol. 845. Springer-Verlag, 1981.

Department of Mathematics, Indiana University, Bloomington, Indiana 47405 (H. B. and C. F.)

Department of Electrical Engineering, University of Minnesota, Minneapolis, MinNESOTA 55455 (A.T.)

Technion, IsRael Institute of Technology, Haifa, Israel (A. T.) 